\title{
Implicit learning in cotton-top tamarins (Saguinus oedipus) and pigeons (Columba livia)
}

\author{
Charles Locurto • Maura Fox • Andrea Mazzella
}

Published online: 12 February 2015

(C) Psychonomic Society, Inc. 2015

\begin{abstract}
There is considerable interest in the conditions under which human subjects learn patterned information without explicit instructions to learn that information. This form of learning, termed implicit or incidental learning, can be approximated in nonhumans by exposing subjects to patterned information but delivering reinforcement randomly, thereby not requiring the subjects to learn the information in order to be reinforced. Following acquisition, nonhuman subjects are queried as to what they have learned about the patterned information. In the present experiment, we extended the study of implicit learning in nonhumans by comparing two species, cotton-top tamarins (Saguinus oedipus) and pigeons (Columba livia), on an implicit learning task that used an artificial grammar to generate the patterned elements for training. We equated the conditions of training and testing as much as possible between the two species. The results indicated that both species demonstrated approximately the same magnitude of implicit learning, judged both by a random test and by choice tests between pairs of training elements. This finding suggests that the ability to extract patterned information from situations in which such learning is not demanded is of longstanding origin.
\end{abstract}

Keywords Implicit learning $\cdot$ Artificial grammar $\cdot$ Cotton-top tamarins $\cdot$ Pigeons

The comparative analysis of learning has identified many parallels between humans and nonhumans, a number of which derive from the study of serial learning. Interest in serial learning is understandable, given that the mechanisms that are

C. Locurto $(\bowtie) \cdot$ M. Fox $\cdot$ A. Mazzella

Department of Psychology, College of the Holy Cross, 1 College St., P.O. Box 75A, Worcester, MA 01610, USA

e-mail: clocurto@holycross.edu invoked in the learning of a list of elements may not only underlie serial learning itself, but may also be involved in other forms of learning and cognition that require an understanding of sequential arrangements, including motor skill learning, numerosity, and language (Brannon \& Terrace, 2002; Terrace, 2010). Previous work has demonstrated that this form of learning in nonhumans reflects characteristics of human performance, such as serial position effects (i.e., better learning of the end elements in a string than of the interior elements: Wright et al., 1985), the organization of elements into chunks (Terrace, 2001; Williams \& Staples, 1992), and the ability to make derived transitive judgments about the elements in a list following serial training (transitive inference, Merritt \& Terrace, 2011; Terrace \& McGonigle, 1994; in rats, Davis, 1992; in corvids, Bond, Kamil, \& Balda, 2003; in rhesus macaques, Rapp, Kansky, \& Eichenbaum, 1996; in pigeons, Wynne, 1997; see Scarf \& Colombo, 2008, for comparative analysis) (See Weaver, Steirn, \& Zentall, 1997, for a noncognitive account of transitive inference performance.) Additionally, a number of lines of evidence indicate that the memorial and representational processes that underlie serial learning are common to humans and nonhumans (D’Amato, 1991).

In one respect, however, the study of serial learning in humans does not have a well-studied parallel in the nonhuman literature. This discrepancy concerns implicit learning, a form of learning in which the acquisition of serial information is not typically accompanied by human subjects' awareness of the experimental contingencies. Implicit learning was first studied in humans by Reber (1967) using a format in which experimental subjects were presented with sets of letter strings that followed a pattern determined by an artificial grammar. Artificial grammars specify arbitrary rules that govern transitions between the members of a set of elements. (See Chomsky, 1957, for the limitations of these grammars as exemplars of language-relevant grammars.) Subjects were asked 
to memorize the strings, but they were not informed about the presence of a pattern. Subjects nevertheless learned these strings more readily than did control subjects who were presented with letter strings that had been composed randomly with respect to the grammar. Additionally, following training, many experimental subjects were unable to articulate the rules of that grammar, an observation in this and other studies that led to the name "implicit" for this type of learning.

Following Reber's (1967) demonstration of implicit learning, numerous additional findings indicated that various forms of patterned information can be acquired implicitly in humans. Some investigators have emphasized that caution must be observed in interpreting these findings. Successful performance may not invariably indicate the acquisition of an abstract pattern or grammar, but may instead be more appropriately interpreted as indicating that subjects have memorized portions of the pattern or recognize similarities between training and the test transitions, rather than acquiring an intimate knowledge of the underlying grammar (e.g., Brooks \& Vokey, 1991). Despite these cautions, interest in this form of learning derives from the fact that a number of human abilities, including language, appear to be learned largely in the absence of explicit instructions. Implicit learning may be a reasonable model of this type of acquisition process (Reber, 1993). Several of these studies have used the artificial grammar design developed by Reber (Clegg, DiGirolamo, \& Keele, 1998; Seger, 1994). A second form of implicit learning, the serial reaction time task (SRT), was developed by Nissen and Bullemer (1987). In their procedure, experimental subjects received repeated presentations of a visual stimulus, an asterisk, on a computer screen. The asterisk's location followed a repeating pattern, and subjects were required to tap an arbitrarily chosen keyboard key when the asterisk appeared at a particular stimulus location. The form followed the pattern DBCACBDCBA, in which each letter referred to one of four spatial locations on the screen where the asterisk might occur. The string was presented without breaks between the end of one presentation of the string and the beginning of the next presentation. The results indicated that the latencies of the experimental subjects were lower than those of control subjects who experienced the same stimuli presented randomly. Furthermore, the experimental subjects often were unable to articulate the nature of the pattern, particularly when the task was made more complex by introducing a second task to be performed concurrently during pattern training (Nissen \& Bullemer, 1987, Exp. 2).

Subsequent work has confirmed Nissen and Bullemer's (1987) basic findings using variations of the initial SRT task. One variation marries the SRT task with Reber's (1967) original artificial-grammar procedure, in that training strings were developed using an artificial grammar. This variation makes the presentation of an individual element probabilistic, given that artificial grammars permit defined variation in terms of which element may occur next in the sequence. Despite this complication, subjects trained with strings generated from an artificial grammar typically demonstrate lower reaction times during training than do subjects trained with randomly constructed strings (Deroost \& Soetens, 2006, Exp. 4; Soetens, Melis, \& Notebaert, 2004).

The position that implicit learning has come to occupy in the study of human cognition makes it valuable to ask whether this form of learning is unique to humans or has comparative origins. It might be argued that there can be no nonhuman analogue of implicit learning, inasmuch as there can be no assessment of subjects' levels of awareness of a pattern, one of the striking features of human implicit learning. In contrast to this argument, Locurto, Gagne, and Levesque (2009) proposed that an implicit learning procedure for a nonhuman would present patterned information to subjects, but reinforcement would be delivered on a random basis, not dependent on learning that pattern, as it is in standard serial learning tasks. The question would then be whether in this format a nonhuman would learn more than was demanded by the experimental contingencies.

Admittedly, any definition of implicit learning is problematic. In the more well-developed literature on human implicit learning, there has been consistent controversy regarding definition, to the extent that at least 12 different definitions have been identified (Frensch \& Rünger, 2003). The definition of implicit learning used here overlaps with the definition of another type of learning studied in human cognition, incidental learning. Incidental learning refers to all forms of learning in human subjects that occur unintentionally - that is, as a consequence of exposure to material, without instructions to learn. In this form of learning, subjects may be able to articulate the nature of the learning when queried following the experiment. We have suggested elsewhere that in the human literature, implicit learning is a subset of these incidental learning procedures in which learning typically occurs without subjects' awareness of the experimental contingencies (Locurto, Gagne, \& Nutile, 2010).

To illustrate this definition of implicit learning in a nonhuman, Locurto et al. (2009) developed a procedure that wedded aspects of the standard SRT task with features taken from standard serial learning procedures, in which reinforcement is delivered only for correct choices. The task, termed implicit chaining, consisted of a five-element chain in which the same visual image was presented repeatedly at different locations $(\mathrm{A} \rightarrow \mathrm{E})$ on a touchscreen. The location presentations followed a pattern, and the subjects, two cotton-top tamarins (Saguinus oedipus), were required to touch the element each time that it appeared in order to advance the chain to the next element. Reinforcement was presented following the fifth element. Following training on the pattern, two types of tests were conducted. In the first type of test, subjects were presented with two elements and were asked to choose one of them 
(e.g., "choose between A and C"). This type of pair-wise testing is standard in serial learning procedures and indicates the extent to which subjects have acquired a sense of serial order. The second test, a random test of the type that is characteristic in SRT studies, presented subjects with a series of five-element chains in which the serial order was randomly determined. The results for the random test indicated that for each tamarin, latencies were higher during the random test than during training, and this finding appeared to be true for each element and each serial position. The results of the pairwise tests indicated that each tamarin chose later elements in the chain rather than earlier elements (e.g., C over A), indicating that they ordered the elements of the chain in terms of their proximity to reinforcement.

Two subsequent series of studies using tamarins have extended this basic procedure (Locurto, Dillon, Collins, Conway, \& Cunningham, 2013; Locurto et al., 2010). These studies revealed that, in the format of presenting elements in an $\mathrm{A} \rightarrow \mathrm{E}$ pattern with reinforcement at the end of the chain, a visually based task in which the elements were defined as different images rather than as different spatial locations of the same image produced approximately the same levels of implicit learning (Locurto et al., 2010, Exp. 1). In this A $\rightarrow E$ format, evidence from pair-wise tests suggested that subjects would choose an element that followed in the chain rather than one that was closer to the end of the chain, and therefore that was presumably of higher associative strength (e.g., choosing $\mathrm{C}$ over $\mathrm{D}$ when this pair-wise test followed the presentation of the first two elements in the chain, $\mathrm{A} \rightarrow \mathrm{B}$ ). This finding suggests that the linkages between elements in the chain were not solely dependent on associative strength, but reflected other forms of association, such as stimulus-stimulus or stimulus-response learning. A subsequent set of experiments indicated that implicit learning took place when such learning was not dependent on reinforcement being presented predictably at the end of the serial chain, but instead was presented randomly across all elements (Locurto et al., 2013, Exps. 1 and 2). Also, implicit learning could be observed when the strings were composed by querying an artificial grammar, rather than by presenting elements in a strict $\mathrm{A} \rightarrow \mathrm{E}$ order (Locurto et al., 2013, Exp. 3).

These findings join a number of other demonstrations using the SRT procedure with nonhumans in which evidence has emerged of decreases in latencies during pattern training and increases during a random test (in rats: Christie \& DalrympleAlford, 2004; Domenger \& Schwarting, 2005; in mice: Christie \& Hersch, 2004; in rhesus macaques: Procyk, Dominey, Amiez, \& Joseph, 2000; in pigeons: Froehlich, Herbranson, Loper, Wood, \& Shimp, 2004, Herbranson \& Shimp, 2008; Herbranson \& Stanton, 2011; in Bengalese finches: Yamazaki, Suzuki, Inada, Iriki, \& Okanoya, 2012). These studies have demonstrated that some learning occurs in nonhumans when reinforcement is randomly presented. We cannot determine from these results whether the observed learning was a function of the same mechanisms across different species. In the literature on serial learning in which reinforcement has been dependent on correct choices, evidence regarding pigeons and monkeys has indicated that these two species may learn serial sequences presented in the typical $\mathrm{A} \rightarrow \mathrm{E}$ order in quite different manners (Terrace \& McGonigle, 1994).

Straub and Terrace (1981) were the first to demonstrate that when pigeons (White Carneaux, Columba livia) acquire an $\mathrm{A} \rightarrow \mathrm{D}$ serial list, they demonstrate in pair-wise tests that the end elements, A and D, are learned about and chosen correctly. However, the interior pair $\mathrm{B} / \mathrm{C}$ is responded to at chance levels, suggesting that the pigeons did not learn the ordinal position of these interior elements, but rather learned exclusively the end-element positioning. Monkeys, in contrast, reliably demonstrate learning of interior elements with correct choices on pair-wise tests (e.g., choosing B over C in a forward serial learning procedure; Chen, Swartz, \& Terrace, 1997; Terrace, 1993, 2001, in Macaca mulatta; D'Amato \& Colombo, 1988, 1990, in Cebus apella). Other differences between pigeons and monkeys have been noted, as well. In pair-wise or subset testing (i.e., three or four elements drawn from a five-element list), the reaction times (RTs) of the responses to the first item of each string provide information as to how pigeons and monkeys understand the ordering of a serial list. For monkeys, RTs to the first item of a pair or subset have been shown to increase as a function of that item's position in the original list. This observation suggests that monkeys scan their memory of the sequence beginning at item A and move serially through the list. In contrast to monkeys, pigeons show similar RTs regardless of the location of the first item's position in the original sequence (Terrace, 2010). Similarly, monkeys show a reliable increase in latencies as the distance between the two members of the tested pair increases, again indicating that they are in some sense scanning their memory of the list, and this process takes longer if elements in the pair are farther apart. For pigeons, the latency to respond in the pair-wise tests is not influenced by this distance effect. These differences indicate that although different species may demonstrate serial learning, the underlying architectures of that learning may not be entirely commensurate (Terrace, 2010).

Given the differences that have been noted between pigeons and monkeys in standard serial learning tasks, in the present work we compared pigeons (Columba livia) with cotton-top tamarins (Saguinus oedipus) on an implicit learning task. In designing the study, we adopted the strategy that the most effective way to understand possible species differences would be to compare two species under conditions in which all facets of the experimental procedure were matched as closely as possible between the species. To that end, we equated as many aspects of the training procedures between 
the two species as was feasible, including the apparatus used, the sequence of reinforcers and training stimuli each session, and the amount of exposure to the implicit task.

The task we chose presented elements in a continuously repeating chain. The element transitions composing the chain were chosen by following an artificial grammar (see Locurto et al., 2013, Exp. 3, for the use of this grammar in a discretetrials procedure). The use of a repeating chain is consistent with the earlier implicit-learning design originated by Nissen and Bullemer (1987). It also captures aspects of experiments designed to assess artificial language learning in infants. In these studies, continuous streams of auditory stimuli that have defined statistical frequencies are presented to subjects. Appreciation of the statistical frequencies is marked by the amount of time that infants attend to familiar and unfamiliar stimulus frequencies during subsequent testing (Saffran, Aslin, \& Newport, 1996). A variety of studies have shown that infants as young as seven months attended longer to words with unfamiliar structures that had been generated from an artificial language than to sentences generated from the same artificial language that possessed familiar structures (Marcus, Vijayan, Rao, \& Vishton, 1999; Saffran, \& Thiessen, 2003). Importantly, from the perspective of the present work, monkeys, like human infants, have also been reported to learn the transitional probabilities between sequences generated from an artificial language and to orient preferentially to unfamiliar transitions during testing (Meyer \& Olson, 2011).

The use of a preconfigured sequence also allowed us to equate the training experiences of both species during each session. That is, we were able to ensure that all element frequencies, transitions between elements, and reinforcement probabilities were equated between species at every point in training. Equating the task in this manner between species was undertaken to provide as clear a picture as possible of the extent to which the two species differed or were comparable in this type of procedure. Previous research has been equivocal with respect to whether primates and pigeons differ consistently across different assays of cognition. A number of studies that have compared primates to pigeons have tended to show that cognitive differences between these species favor more complexity in performance in primates (Roberts, 2010, with numerical magnitude estimation; D'Amato, Salmon, Loukas, \& Tomie, 1986, with identity and conditional matching; Smith et al., 2012, with dimensional analysis and the acquisition of category rules). Yet, there have also been a number of demonstrations of equivalent performance between primates and pigeons in other complex tasks (Roberts \& Mazmanian, 1988, with some forms of abstract concept learning; Wright \& Katz, 2007, 2009, with the acquisition of same vs. different concepts; Colombo, Cottle, \& Frost, 2003, with delayed matching to sample). Perhaps the most impressive of these demonstrations has been the recent finding that pigeons' ability to employ abstract numerical rules can be shown to be indistinguishable from that displayed by monkeys (Scarf, Hayne, \& Colombo, 2011).

Given that comparisons between primates and pigeons on cognitive assays have yielded a mixed picture, our hypotheses were generated not from that literature, but from a consideration of how implicit learning has been characterized in the human cognition literature, and how it may differ from standard explicit learning - that is, learning in which human subjects can report consciously on the nature of the task. Perhaps the principal feature of implicit learning that has drawn attention is the fact that it occurs typically, though not universally, without conscious awareness. Reber (1993) has argued that this feature of implicit learning may indicate that it should be robust with respect to disruptions owing to various psychological disorders, due to the fact that unconscious processes evolved earlier in the evolutionary timeline than conscious processes (see also Dienes \& Berry, 1997). Evidence of this effect has come from studies indicating that at least some forms of implicit learning, including SRT tasks, are conserved in cases of closed-head injury (CHI) and amnesia-disorders in which consciousness may be impaired - whereas explicit learning is diminished (Knowlton \& Squire, 1994; Nissley \& Schmitter-Edgecombe, 2002).

From this quality of robustness, Reber (1993) further suggested that implicit learning should evidence the quality of commonality, meaning in this sense that implicit learning, since it possesses a much longer evolutionary history than explicit learning, should show uniformity across different nonhuman species. To the extent that this proposition is valid, we anticipated that tamarins and pigeons would produce equivalent performance on this implicit task in terms of acquisition, exposure to a random test, and pair-wise testing in which two-item choices were presented to subjects following acquisition.

\section{Method}

\section{Subjects}

Tamarins These subjects were three male cotton-top tamarins: Fergus was eight years old at the start of the study, Homer was seven years old, and Windsor was 19 years old. All three subjects had served previously in implicit-learning experiments (viz. Locurto et al., 2013; Locurto et al., 2009; Locurto et al., 2010). The tamarins were born and housed individually at the New England Primate Research Center, Southborough, Massachusetts, and were maintained under the guidelines of the Committee on Animals of the Harvard Medical School. Daily feeding consisted of a morning feeding and a second one in the afternoon. On training days, they received their morning feeding for approximately $2 \mathrm{~h}$. Feedings consisted of monkey chow supplemented with fresh 
fruit. The food was removed $3-4 \mathrm{~h}$ prior to training, which took place in the early afternoon. The tamarins received their afternoon feeding immediately following a session. With this schedule, each tamarin was maintained at about $95 \%$ of its free-feeding weight. Training was conducted on average four times each week.

Pigeons The three pigeon subjects were Silver Kings (Columba livia), approximately 3-5 years old at the start of the study, obtained from the Palmetto Pigeon Plant, Sumter, South Carolina. They were each experimentally naïve at the start of training. The pigeons were housed individually in cages that measured $50 \mathrm{~cm}$ on each side. The cages were custom designed to be large enough to allow the pigeons to engage in species-typical behaviors such as perching, flapping their wings, and moving about the cage. The cages were constructed with wire mesh on each side, thereby allowing adjacent pigeons to be in visual contact with each other (Alternative Design Mfg., Siloam Springs, AR).

The tamarins were run first; the pigeons were run after the end of the tamarins' training and testing. We matched the pigeons' training parameters as closely as possible to those of the tamarins. To that end, the pigeons were maintained at $95 \%$ of their free-feeding weight to match the deprivation levels characteristic of the tamarin sample during their training. Free water was provided in the cages, and weights were maintained by feeding the pigeons approximately $20 \mathrm{~g}$ of food following experimental sessions. Sessions were run 4-5 days each week.

\section{Stimuli and apparatus}

The experiment was programmed using Superlab (4.07, Cedrus). The stimulus for both species consisted of an image of a set of whiskey barrels of approximately $200 \times 200$ pixels, displayed against a white background on a touchscreen (ELO CarrollTouch infrared; Tyco Electronics, Berwyn, PA; screen resolution: $800 \times 600$ pixels). The screen was $38.1 \mathrm{~cm}$ on the diagonal. For both species, the front of the touchscreen was fitted with a clear Plexiglas bezel that allowed access to the touchscreen at six locations $(10 \times 10 \mathrm{~cm}$ holes $)$ arranged in a 2 $\times 3$ array across the touchscreen. The image was displayed in approximately the center of each location against a white background. All of the subjects received the same image locations for elements $\mathrm{A} \rightarrow \mathrm{D}$. Reinforcement consisted of a $97-$ $\mathrm{mg}$ pellet for both species. For tamarins, the pellet was composed of chocolate sucrose. For pigeons, the pellet was composed of mixed grain (Test Diet, Purina Mills, LLC). The reinforcer was delivered into a dish located to the side of the touchscreen via a rotary pellet feeder (Coulbourn Instruments, Whitehall, PA). Each response produced an auditory stimulus approximately $100 \mathrm{~ms}$ in duration that was the default auditory signal produced by the monitor.
Grammatical sequences The finite-state grammar that was used to generate the training sequence is displayed in Fig. 1. The four elements of the grammar (A, B, C, D) each represent a spatial location on the touchscreen. For each element, there were two grammatical transitions and two nongrammatical transitions. For element A, the grammatical transitions were $\mathrm{A}$ and $\mathrm{D}$, and the nongrammatical transitions were $\mathrm{B}$ and $\mathrm{C}$. For element $\mathrm{B}$, the grammatical transitions were $\mathrm{A}$ and $\mathrm{D}$, and the nongrammatical transitions were $\mathrm{B}$ and $\mathrm{C}$. For element $\mathrm{C}$, the grammatical transitions were $\mathrm{B}$ and $\mathrm{C}$, and the nongrammatical transitions were $\mathrm{A}$ and $\mathrm{D}$. For element $\mathrm{D}$, the grammatical transitions were $\mathrm{B}$ and $\mathrm{C}$, and the nongrammatical transitions were $\mathrm{A}$ and $\mathrm{D}$. The grammatical sequence used in baseline training was constructed so that each of the four elements was equally represented in the complete sequence $(n=$ 32 representations), as was each grammatical transition ( $n=$ 8). One caveat to this probabilistic sequencing was that two of the elements in this grammar, A and C, could be followed by themselves, with no logical constraints on how many such repetitions could occur sequentially. We adopted the rule that elements $\mathrm{A}$ and $\mathrm{C}$ could occur up to four times consecutively. We also balanced the reinforcement for each element $(p=.16$ for the first 12 sessions, $p=.12$ thereafter and during all test sessions) and for each grammatical transition. The result was a chain of 128 elements. Each session began at a different point in the chain, and the distribution of reinforcements was altered each session. We matched the pigeons' training sequences each session with the sequences experienced by the tamarins during their corresponding session. The result was that both species experienced the same sequences and the same distribution of reinforcers each session.

\section{Procedure}

The tamarins were tested in their home cages. The touchscreen was placed in front of the subject's home cage by using a load lifter (Genie Industries, Redmond, WA). The front door to the animal's cage was then opened, allowing the subject access to the touchscreen and food dish. The pigeons were instead transported to a test chamber that was constructed of clear Plexiglas, $30 \times 30 \mathrm{~cm}$ by $33 \mathrm{~cm}$. The front of the test chamber was open and fitted against the same touchscreen used for the

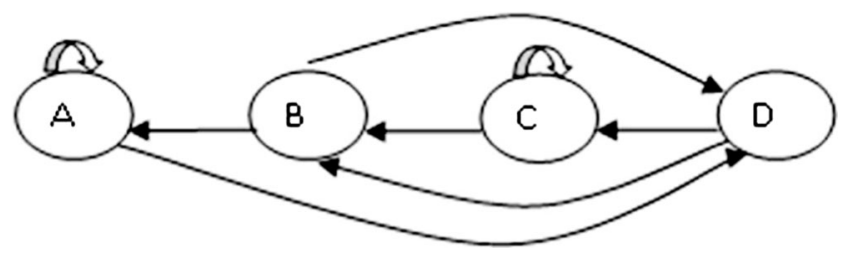

Fig. 1 Finite-state grammar used to generate the sequence of elements used during all baseline training. In this grammar, there are two grammatical transitions and two nongrammatical transitions for each element (e.g., for element B, A and D represent grammatical transitions, whereas $\mathrm{B}$ and $\mathrm{C}$ represent nongrammatical transitions) 
tamarins. Free water was provided in the chamber, which was fitted into an isolation cubicle (Coulbourn Instruments, Whitehall, PA).

The first touch to an element advanced the chain to the next element without delay. Touches to blank areas of the screen were recorded but had no consequences. If reinforcement was programmed for an element, a screen touch of the element changed the image to one with a blue border for approximately $1 \mathrm{~s}$, concurrent with the delivery of a reinforcer. Following the blue-bordered image, the screen was blank (white) for $1 \mathrm{~s}$. Following the 1-s blank screen, the sequence continued.

Baseline training Since the tamarins were experienced, we presented them on the first day of training with a full session of exposure to the grammatical chain. For purposes of quantifying the amount of training experienced each session, and for identifying where in the sequence sessions should begin and end, we arbitrarily divided the 128 -element sequence into segments, such that one full presentation of the sequence equaled 25 segments. Each segment consisted of from three to seven elements. We began each session at a different segment. Sessions for the tamarins terminated after $50 \mathrm{~min}$. At this session duration, the tamarins completed on average 40 segments per session. Training proceeded for the tamarins until each subject had reached a stability criterion, defined as completing at least 15 sessions of training such that in four of the last five sessions, the median latency was within $\pm 15 \%$ of the median latency across those sessions. Latency was used as the stability criterion because there were no errors, in the sense of incorrect choices, in this procedure. Hence, criteria based on correct performance were not possible.

Windsor met the baseline stability criterion in 22 sessions, Fergus in 24 sessions, and Homer in 59 sessions. The larger number of sessions for Homer than for the other two tamarins resulted from the fact that the study was begun in a summer research period that lasted nine weeks. By the end of that period, Fergus and Windsor had finished the experiment, but Homer, who had received 39 sessions during this period, had not met the stability criterion. More than four weeks passed before Homer resumed training. He received 20 sessions during this second training period before he met the stability criterion.

The pigeons were experimentally naïve at the start of training and were given a number of sessions of shaping prior to beginning exposure to the grammatical sequence. They received four feeder training sessions, followed by 14 sessions in which the training stimulus was presented in a fixed location in the center of the touchscreen on a fixed-ratio-1 schedule, such that each response was reinforced. We judged the pigeons' responding to be stable at this point, so training was begun following this exposure sequence, and the pigeons received the same sequence of stimuli and reinforcements as did the tamarins during each session. Sessions were scheduled for
$50 \mathrm{~min}$ as they had been for the tamarins, but the pigeons produced significantly lower latencies than did the tamarins and virtually no errors, resulting in the pigeons receiving many more segments within the 50-min sessions than had the tamarins (average for 50 -min sessions: tamarins, 40 segments; pigeons, 132 segments). After the sixth session with this number of segments completed, we limited the pigeons to 30 segments per session for the next 20 sessions. This restriction resulted in the pigeons receiving approximately the same total number of baseline segments $(n=1,392)$ as had the tamarins $(n=1,400)$ by the end of training. To experience this number of segments, the tamarins were run for 35 sessions on average, the pigeons for 26 sessions.

Given that different segments of training might contain different frequencies of the four elements in the grammar, the baseline data for both species were assembled for analysis by using the following procedure: We first arranged each session's data in four columns, one column for each element. We then considered one row of $\mathrm{ABCD}$ latencies or errors to constitute one data unit. We used Data Units 11-30 for each subject during the first five and last five sessions for the analysis of baseline performance. This procedure yielded a sample of 100 latency and 100 error data units for the first five sessions and the last five sessions. The practice of constructing data units in this manner led to larger degrees of freedom $(d f)$ for the baseline and random-test analyses than would using the subjects themselves as data units.

Pair-wise tests Following baseline training, pair-wise testing began. Each pair-wise test session contained between 50 and 60 segments. During all segments, reinforcement was delivered according to the same schedule that had been in effect during baseline training. Following a period of baseline training that lasted between four and eight segments, the pair-wise tests were begun. During a pair-wise test segment, the sequence was stopped at a predetermined point, and two elements were presented simultaneously. The two elements were chosen from a set of three possible elements. One of these was termed "Next," meaning that this element was the next element in the sequence after the last element presented before the pair-wise choice. A second element was termed "Grammatical," meaning that it was a grammatical transition from the last item presented in the sequence, but it was not the scheduled next element. The choice of a grammatical element in the pair-wise test was therefore dictated by which element came next in the sequence. The third element was termed "Nongrammatical," meaning that it was a nongrammatical transition from the last element presented in the sequence before the pair-wise choice. The choice of this element was made randomly from the two Nongrammatical elements that were possible transitions for a given element. The frequencies with which Nongrammatical elements were used were equated across all pair-wise tests. The first response to either 
element in a pair-wise choice ended the segment. Reinforcement was scheduled randomly following choices with the same probabilities used during baseline training. A subject's particular choice did not determine whether reinforcement would be delivered. Each test session consisted of six to seven pair-wise tests interspersed with baseline segments. Each subject nominally received 20 pair-wise tests for each of the three types of pairings (Next-Grammatical, Next-Nongrammatical, Grammatical-Nongrammatical), for a total of 60 pair-wise tests spread across ten sessions.

Random test The random-test session followed the last pairwise test session. In this session, the first 25 segments were baseline segments. For the next nine segments, or 56 elements, the elements were presented in randomized order, with reinforcement delivered with a probability of .12 following an element, as had been done during baseline. During the random test, $81 \%$ of the transitions between elements were nongrammatical. For analysis, the baseline performance was matched to the random test in terms of element frequencies. The baseline elements were chosen by working backward from the last grammatically sequenced elements prior to initiation of the random test. The 56 elements used during the random test yield 54 degrees of freedom if a subject completed all random-test elements.

Since the random test served as global assessment of whether learning in any form had occurred, we present the results of this test before the results of the pair-wise tests, which are more fine-grained assays of what had been learned.

Data analyses The principal dependent measure was latency. Errors, defined as touchscreen responses that were outside the area designated by one of the images, constituted a second measure. Choices during the pair-wise tests constituted a third measure. The analysis of latencies focused on differences between the baseline and random segments in the random-test session. As is detailed in the Results section, the latencies failed to meet homogeneity-of-variances criteria for correlated samples using the Pitman-Morgan test (Kenny, 1953). This failure occurred during baseline training in analyses of the first five versus last five sessions, as well as during the random test in the comparison of baseline to the random-test performance. Therefore, latencies were log-converted prior to analysis. The logged latencies also failed the Pitman-Morgan test in a number of comparisons. Therefore, all latency analyses were accomplished using the logged latencies and nonparametric statistics. For tests between latencies obtained from the same subject, we used the nonparametric Wilcoxon signed-rank test, and the results are reported in terms of the $Z$ distribution. All pair-wise test results were evaluated using the sign test. Where possible, we followed the convention of reporting exact alpha levels, with the exception that probabilities below .001 were reported as $p<.001$. The results of some nonparametric tests are reported in terms of categorical $p$ levels- $.05, .01$, and .001 -instead of exact probability levels.

The SKEW function in Excel (2010) indicated positive skew for the samples of tamarins $($ SKEW $=0.74)$ and pigeons $(\mathrm{SKEW}=1.10)$ for the logged latencies during baseline training. During the random test for baseline versus random-test segments, there was moderate skewness for the pigeons $($ SKEW $=0.72)$ but not for the tamarins $($ SKEW $=0.08)$. Bulmer (1979) offered the following rule of thumb regarding skewness: If skewness is less than -1 or greater than +1 , the distribution is highly skewed. If it is between -1 and $-1 / 2$ or + $1 / 2$ and +1 , the distribution is moderately skewed, and if skewness is between $-1 / 2$ and $+1 / 2$, the distribution is approximately symmetric. According to this metric, the latency distributions in this study were on average moderately skewed. This characteristic led us to use medians to represent the average performance during baseline training and the random test.

The use of medians encouraged the use of median-based dispersion measures. We report two such measures that are each less influenced by extreme values than their meanbased counterparts. In place of the $95 \%$ confidence interval measure, which is mean-based, we adopted a similar measure that is median-based, the $95 \%$ confidence interval for the median (CIM). CIM is calculated by rank-ordering all latencies for a given treatment and then applying the following formula to determine the ranks of the relevant latencies: ${ }^{1}$

$n / 2 \pm 1.96 \sqrt{n / 2}$

We also adopted a median-based measure to replace the standard deviation - the median absolute deviation (MAD), the formula for which is ${ }^{2}$

$\operatorname{median}\left(\left|x_{i}-\operatorname{median}\left(x_{i}\right)\right|\right)$.

To our knowledge, there are no agreed-upon nonparametric assessments of effect size (see Wuensch, 2012). We used Cohen's $d$ statistic on the logged latencies and raw errors for assessing the effect sizes of the baseline training differences (first five vs. last five sessions) and between the baseline and random-test differences. We followed the suggested practice of using original standard deviations rather than paired variances or pooled standard deviations for calculations involving correlated samples (see Becker, 2000).

\footnotetext{
${ }^{1}$ This formula was obtained from the Statistics and Research Methodology website: https://epilab.ich.ucl.ac.uk/coursematerial/ statistics/non_parametric/confidence_interval.html, downloaded 12.28. 12.

${ }^{2}$ This formula was taken from http://en.wikipedia.org/wiki/Median absolute_deviation, downloaded 1.2.13.
} 


\section{Results}

Baseline

Table 1 presents all tests of homogeneity of variances using the Pitman-Morgan formula for baseline training and for the random test. Figure 2 shows the median latencies during the first five and last five baseline sessions for each tamarin and each pigeon. Each tamarin as well as the combined sample of tamarins failed Pitman-Morgan for the raw latencies. Two of the three subjects, Homer and Windsor, passed PitmanMorgan using the logged latencies, whereas Fergus still failed Pitman-Morgan, as did the combined sample. Two of the three tamarins, Fergus and Homer, produced significantly shorter latencies during the last five sessions than during the first five sessions, whereas Windsor did not show evidence of latency reductions as a function of training (Wilcoxon test/Cohen's $d$ : Fergus, $Z=5.25, p<.001, d=0.80$; Homer, $Z=6.08, p<.001, d=1.04$; Windsor, $Z=0.95, p=.17, d=$ $0.17)$. The latencies for the combined sample of tamarins also showed significant reductions in latency $(Z=7.75, p<.001$, $d=0.65$ ). The $95 \%$ median confidence intervals (CIM) were composed of lower latencies and smaller bandwidth during the last five sessions than during the first five sessions for each tamarin and for the combined sample (Fergus: first five, 2, 871-3,877 ms; last five, 1,751-2,336 ms; Homer: first five, 6,473-9,120 ms; last five, 2,901-3,865 ms; Windsor: first five, 2,027-2,589 ms; last five, 1,882-2,367 ms; combined sample: first five, 3,109-4,019 ms; last five, 2,232-2,560 ms).

Two of the three tamarins, Fergus and Homer, and the combined sample also showed significant reductions in errors during the last five sessions relative to the first five sessions (Fergus: $Z=3.93, p<.001, d=0.59$; Homer: $Z=4.05, p<$ $.001, d=0.66$; Windsor: $Z=-1.83, p=.03, d=-0.28$; combined sample: $Z=3.21, p<.001, d=0.23$ ). Errors were defined as contacts with portions of the touchscreen that were outside the area defined as the stimulus. Windsor showed a rise in errors between the first five and last five sessions, from 1.29 errors/segment during the first five sessions to 1.52 errors/segment during the last five sessions. For the combined tamarin sample, errors averaged 0.99/segment during the first five sessions and $0.81 /$ segment during the last five sessions. Average errors were represented using means and not medians, given that a large proportion of the data units contained no errors.

Figure 2 (bottom panel) gives the median baseline latency data for the pigeons. Analysis of the raw latencies indicated that all three pigeons and the combined sample failed PitmanMorgan. All three pigeons also failed Pitman-Morgan for the logged latencies, but for the combined sample, the logged latencies did pass Pitman-Morgan. The combined sample showed reductions in latencies across training, although this trend was significant individually only for Pigeon $2(Z=7.46$, $p<.001, d=1.44$; Pigeon $3, Z=-0.60, p=.27, d=0.00$; Pigeon $4, Z=-1.21, p=.11, d=0.00$; combined sample, $Z=$ $3.71, p<.001, d=0.32$ ). Additionally, only Pigeon 2 and the data for the combined sample showed lower-magnitude confidence intervals and reduced bandwidth during the last five relative to the first five sessions (Pigeon 2: first five, 2,141-2, $513 \mathrm{~ms}$; last five, 1,364-1,516 ms; Pigeon 3: first five, 1,5391,841 ms; last five, 1,509-1,821 ms; Pigeon 4: first five, 1, 877-2,145 ms; last five, 1,866-2,165 ms; combined sample: first five, 1,346-2,621 ms; last five, 1,609-1,770 ms). For pigeons, error rates were low from the start of training and were virtually identical in magnitude across all baseline sessions. Errors averaged 0.10/segment during the first five sessions and 0.11 /segment during the last five sessions $(Z=-0.60, p=.27, d=-0.04)$. Only Pigeon 2 showed

Table 1 Homogeneity of variance tests for baseline and random test

\begin{tabular}{|c|c|c|c|c|}
\hline & \multicolumn{2}{|l|}{ Baseline } & \multicolumn{2}{|l|}{ Random Test } \\
\hline & Raw Latencies & Logged Latencies & Raw Latencies & Logged Latencies \\
\hline \multicolumn{5}{|l|}{ Tamarins } \\
\hline Fergus & $t(98)=15.58^{* * *}$ & $t(98)=3.50^{* * *}$ & $t(54)=0.14, p=.88$ & $t(54)=0.18, p=.86$ \\
\hline Homer & $t(98)=5.16^{* * *}$ & $t(98)=1.26, p=.21$ & $t(54)=8.35^{* * *}$ & $t(54)=0.23, p=.81$ \\
\hline Windsor & $t(98)=3.23^{* * *}$ & $t(98)=0.38, p=.70$ & $t(54)=6.26^{* * *}$ & $t(54)=1.50, p=.14$ \\
\hline Tamarins combined & $t(298)=14.78^{* * *}$ & $t(298)=6.36^{* * *}$ & $t(166)=13.11^{* * *}$ & $t(166)=0.65, p=.52$ \\
\hline \multicolumn{5}{|l|}{ Pigeons } \\
\hline 2 & $t(98)=21.32^{* * *}$ & $t(98)=7.43^{* * *}$ & $t(54)=3.23, p=.002$ & $t(54)=1.50, p=.13$ \\
\hline 3 & $t(98)=5.93^{* * *}$ & $t(98)=3.52^{* * *}$ & $t(54)=2.71, p=.01$ & $t(54)=5.85^{* * *}$ \\
\hline 4 & $t(98)=21.74^{* * *}$ & $t(98)=5.73^{* * *}$ & $t(54)=30.61^{* * *}$ & $t(54)=1.36, p=.17$ \\
\hline Pigeons combined & $t(298)=7.38^{* * *}$ & $T(298)=0.03, p=.76$ & $t(164)=38.38^{* * *}$ & $t(164)=3.31^{* * *}$ \\
\hline
\end{tabular}

Significant $t$ ratios indicate that the comparison failed Pitman-Morgan. ${ }^{* * *} p<.001$; all other alpha levels are reported as exact probabilities 

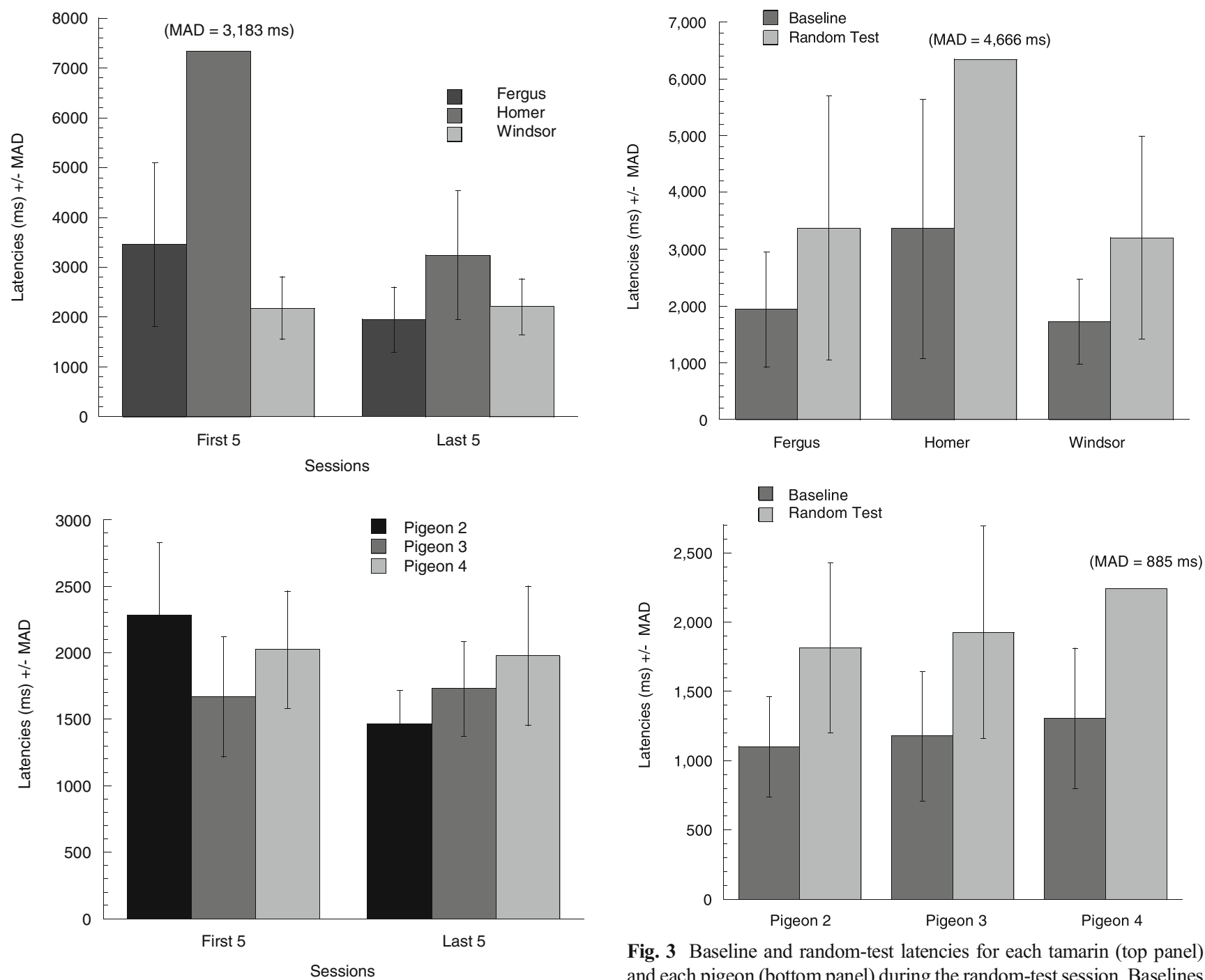

Fig. 3 Baseline and random-test latencies for each tamarin (top panel) and each pigeon (bottom panel) during the random-test session. Baselines were matched to the random-test sequences in terms of element frequencies. Baseline elements were chosen by working backward from the last grammatically sequenced elements prior to initiation of the random test until equal frequencies of each element were obtained, in comparison to the random test

a significant reduction in errors across training $(Z=2.70, p=$ $.003, d=0.43$; Pigeon $3: Z=-0.57, p=.27, d=-0.12$; Pigeon $4: \mathrm{Z}=-1.58, p=.057, d=-0.27)$.

Comparisons of baseline performance between the tamarins and pigeons indicated that the pigeons produced significantly lower latencies and fewer errors than did the tamarins during both the first five and last five sessions (latencies: first five, $Z=10.99, p<.001, d=1.05$; last five, $Z=9.15, p<.001$, $d=0.75$; errors: first five, $Z=15.57, p<.001, d=1.58$; last five, $Z=12.19, p<.001, d=1.15$ ).

\section{Random test}

Figure 3 shows the results of the random test for the tamarins and pigeons, presented in terms of median latencies during the baseline and subsequent random element presentations during

the random-test session. As can be seen in Table 1, for the combined sample of tamarins, the raw latencies did not pass Pitman-Morgan. Individually, the latencies for both Homer and Windsor did not pass Pitman-Morgan, whereas the latencies for Fergus did show homogeneity of variances. The logged latencies for the combined sample did pass PitmanMorgan, as did the latencies for each subject.

Wilcoxon tests on the baseline versus random latencies revealed that the random-test latencies for each tamarin individually and for the combined sample were higher than those at baseline (Fergus: $Z=1.63, p=.05, d=0.32$; Homer: $Z=$ $2.33, p=.009, d=0.45$; Windsor: $Z=2.75, p<.001, d=0.58$; combined sample: $Z=3.91, p<.001, d=0.44)$. For the combined sample, there was on average a $78 \%$ increase in latencies during the random test, as compared to baseline 
(individually: Fergus, $73 \%$; Homer, $89 \%$; Windsor, $85 \%$ ). It was also the case that for each subject and for the combined sample, the confidence intervals were elevated in absolute value and were of broader bandwidth during the random test than at baseline (Fergus: baseline, 1,596-2,704 ms; random test, 2,020-4,555 ms; Homer: baseline, 2,212-4,712 ms; random test, 4,154-9,307 ms; Windsor: 1,366-2,160 ms; random test, 2,343-4,149 ms; combined sample: baseline, 1,824-2, $614 \mathrm{~ms}$; random test, 2,967-4,691 ms).

We observed no significant error differences between baseline and the random test for any subject individually or for the combined sample (Fergus: $Z=1.42, p=.08, d=0.25$; Homer: $Z=0.27, p=.39, d=0.07$; Windsor: $Z=-0.37, p=.36, d=$ 0.03 ; combined sample: $Z=0.99, p=.16, d=0.09$ ). Tamarins averaged 1.24 errors/segment $(S D=1.49)$ during baseline and 1.34 errors/segment $(S D=1.48)$ during the random test.

Figure 3 (bottom panel) gives the random-test results for the pigeons. As is noted in Table 1, for each pigeon and for the combined sample, the raw latencies did not pass PitmanMorgan. For the logged latencies, Pigeons 2 and 4 passed Pitman-Morgan, whereas Pigeon 3 and the combined sample did not.

The comparison of the baseline and random-test latencies revealed that the random-test latencies were reliably higher than the baseline latencies for each subject individually and for the combined sample (Pigeon 2: $Z=3.49, p<.001, d=$ 0.67; Pigeon 3: $Z=3.52, p<.001, d=0.69$; Pigeon $4: Z=$ $3.19, p<.001, d=0.74$; combined sample: $Z=5.88, p<.001$, $d=0.71)$. The percent increase in latencies for the combined sample was $56 \%$ (Pigeon 2, $65 \%$; Pigeon 3, $64 \%$; Pigeon 4, $72 \%$ ). The $95 \%$ confidence intervals (CIM) were of lower magnitude and of more narrow bandwidth during baseline than in the random test (Pigeon 2: baseline, 901-1,175 ms; random test, 1,299-1,846 ms; Pigeon 3: baseline, 893-1, $330 \mathrm{~ms}$; random test, 1,209-1,964 ms; Pigeon 4: baseline, 1,000-1,605 ms; random test, 1,769-2,589 ms; combined sample: baseline, 1,075-1,426 ms; random test, 1, $780-2,155 \mathrm{~ms}$ ).

Our analysis of errors between baseline and the random test revealed no differences in errors for the individual subjects, but there was a reliable difference in errors between baseline and the random test for the combined sample (Pigeon 2: $Z=$ $1.40, p=.08, d=0.27$; Pigeon $3: Z=1.45, p=.07, d=0.30$; Pigeon $4: Z=1.18, p=.12, d=0.20$; combined sample: $Z=$ $2.33, p=.009, d=0.21)$. Errors averaged $0.13 /$ segment $(S D=$ 0.35 ) during baseline and 0.30 /segment $(S D=1.08)$ during the random test.

Pigeons produced lower latencies than did tamarins, both during the baseline period $[t$ test assuming unequal variances: $t(168)=4.68, p<.01, d=0.51]$ and during the random test $[t(182)=3.48, p<.01, d=0.38]$. Pigeons also produced significantly fewer errors than did tamarins during baseline [ $t$ test assuming unequal variances: $t(188)=9.06, p<.01, d$
$=0.99]$ and the random test $[t(317)=7.21, p<.01, d=0.79]$. The comparison of random-test performance between the tamarins and pigeons was also accomplished by analyzing the difference scores between the baseline and random-test latencies. This analysis was conducted by pairing each random-test element with its comparable baseline element and computing a difference score between them for each element presentation and each subject. Each difference score was then normalized against its baseline [i.e., (baseline - random test)/baseline], to take into account differences in absolute latency levels between the subjects and species. The median normalized difference scores were 0.46 for tamarins and 0.57 for pigeons. No reliable between-species differences emerged in the normalized differences scores (Mann-Whitney $U: Z=.22$, $p=.41)$.

Pair-wise tests

Figure 4 gives the choice proportions of the first element in each pair-wise test for the tamarins and pigeons. For the first pair-wise test, Next versus Nongrammatical elements, the proportion displayed in the figure is the proportion choice of Next elements. Fergus and Windsor completed between 57 and 60 pair-wise tests. Homer completed a total of 29 pair-wise tests, at least six of each comparison. The tamarins reliably chose Next over Nongrammatical elements (sign test: $p=.002$ ). In the choice between Grammatical and Nongrammatical elements, the choice of Grammatical elements approached significance $(p=.07)$. The tamarins also reliably chose Next over Grammatical elements $(p=.02)$, although two of the three tamarins, Fergus and Windsor, were indifferent in their choices of Grammatical and Next elements. Homer showed a strong preference for Next elements (13 out of 16 choices).

The comparable data for pigeons are also given in Fig. 4 (bottom panel). All pigeons completed between 59 and 60 pairwise tests. The pigeons' choice of Next over Nongrammatical elements approached significance $(p=.058)$, and they reliably chose Grammatical over Nongrammatical elements $(p=.03)$. The pigeons were indifferent between Next and Grammatical elements (31 out of 60 choices).

To determine whether the tamarins and pigeons performed similarly on the pair-wise tests, we conducted a 2 (species) $\times 3$ (pair-wise tests) mixed-model analysis of variance (using SPSS, v. 22). The results indicated no significant difference between species on any pair-wise test, $F(1,4)=0.084, p>.10$, and no significant Species $\times$ Pair-Wise Test interactions, $F(1,4)>.10$ for each test. Additionally, error variances did not differ for any pair-wise test using Levene's test for the equality of error variances (SPSS, v. 22). Since the tamarins and pigeons performed similarly on the pair-wise tests, we combined the two samples into one analysis for each pairwise comparison. For this combined-species sample. Next 

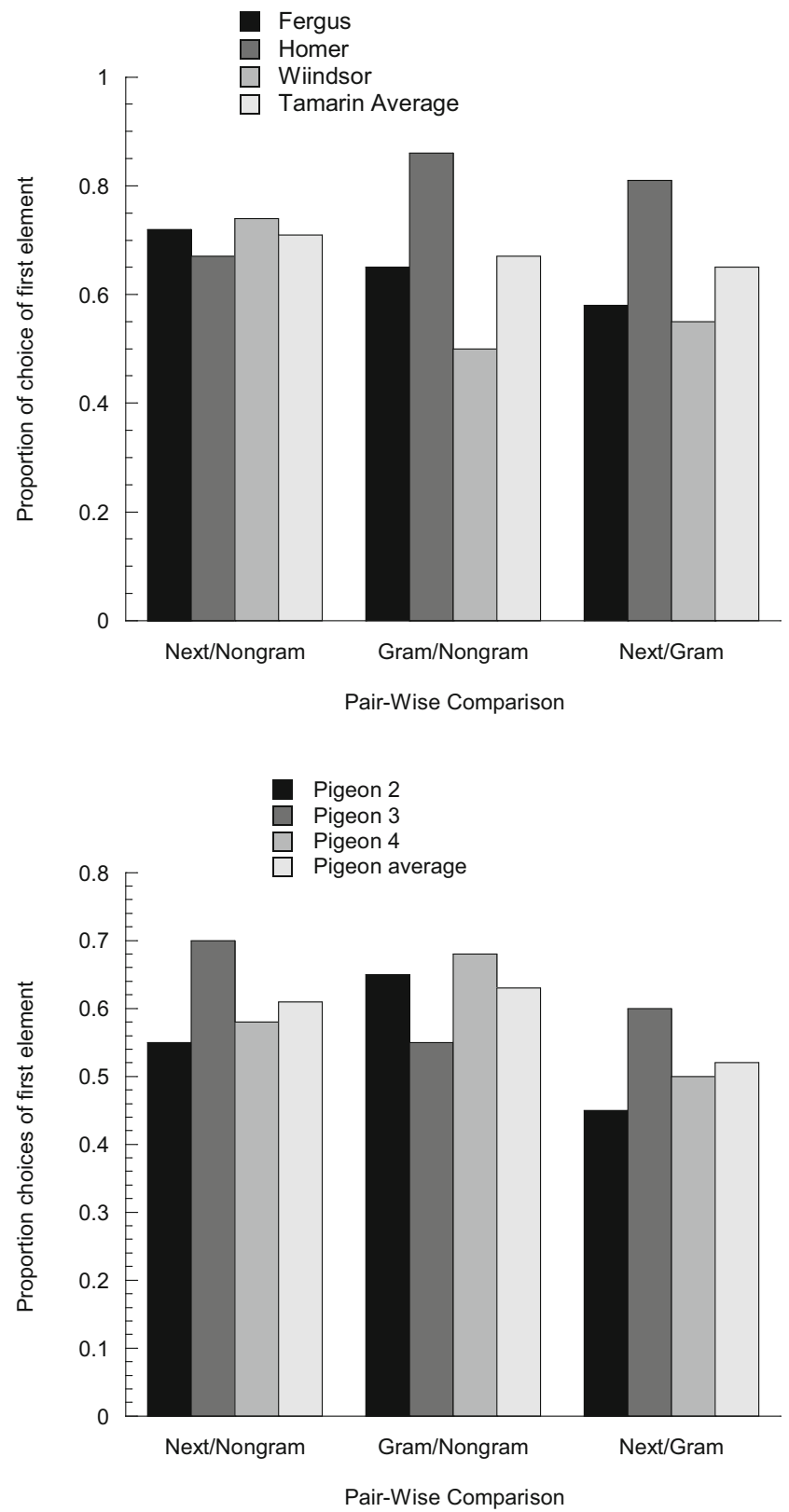

Fig. 4 Proportions of choices of the first element in all pair-wise test pairs for each tamarin (top panel) and each pigeon (bottom panel). "Next" elements were the grammatical elements that were scheduled to be the next element in the baseline sequence. "Grammatical" elements constituted a grammatical transition from the last item presented in the sequence prior to the pair-wise test, but they were not the next scheduled element. "Nongrammatical" elements represented a nongrammatical transition from the last element presented in the sequence before the pair-wise choice

elements were chosen reliably over Nongrammatical elements $(p<.001)$, and Grammatical elements were also chosen reliably over Nongrammatical elements $(p=.007)$. For the combined-species sample, subjects chose Next elements over Grammatical elements, although this comparison only approached significance $(p=.07)$.

\section{Discussion}

The principal finding of this study was that pigeons and tamarins were comparable in their implicit learning of a repeating sequence in which elements were chosen by following an artificial grammar. Performance on the random test indicated similar levels of disruption, suggesting in turn similar magnitudes of learning. Tamarins exhibited a $78 \%$ increase in latencies during the random test as compared to baseline, whereas pigeons showed a $56 \%$ increase in latencies during this test. The comparison of normalized baseline/random-test difference scores found no reliable differences between the species. These similarities in performance were observed despite the fact that the tamarins had participated in several previous experiments in implicit learning, whereas the pigeons were experimentally naïve. The pigeons adjusted to the experimental contingencies within the first few sessions and, as we noted in the Method section, we had to limit the number of segments experienced by the pigeons within a session so as to match the overall training parameters experienced by the tamarins. Two of the three pigeons ( 3 and 4 ) reached asymptotic levels of baseline performance during the first five sessions and displayed no reductions in latencies or errors between the first five and last five sessions. This rapid adjustment of the pigeons to the experimental contingencies should perhaps not be surprising, given the highly prepared nature of the pigeons' visual stimulus $\rightarrow$ peck $\rightarrow$ food response system, a feature that figures prominently in accounts of pigeons' autoshaping performance, particularly the persistence of responding in the face of an omission contingency (negative automaintenance; see, e.g., Killeen, 2003; Locurto, Terrace, \& Gibbon, 1981).

The pair-wise tests also yielded evidence of similar performance between the species, although the results of the pairwise tests were not as uniform either within or between species as were the random-test results. It should be kept in mind that the different pair-wise tests assay different aspects of performance. The choice between Next and Nongrammatical elements essentially asks whether the subject has learned anything about the element sequences. That is, the choice of Next elements over Nongrammatical elements might be made either on the basis of the learning of specific transitions or through some appreciation of the grammar, since Next elements were also grammatical. Both tamarins and pigeons showed a preference for Next over Nongrammatical elements, although the pigeons' preference only approached significance $(p=.058)$. For the combined sample of both species, Next elements were reliably chosen over Nongrammatical elements. This test essentially addressed the same question that was addressed by the random test. These two sources of evidence converge on the conclusion that both species demonstrated that something was learned about the sequence, and that this learning appeared to be approximately comparable in magnitude between the two species. 
In terms of learning something about the grammar, the more interesting comparison was between Grammatical and Nongrammatical elements. Neither element in this choice test was the element that came next at the point in the sequence at which the test was administered. If a subject had learned nothing about the sequence or had simply memorized aspects of the sequence, the subject should show indifference between the elements in this comparison. If the subject had some understanding of aspects of the grammar, it should choose Grammatical elements. Both the tamarins and pigeons preferred Grammatical elements, although the tamarins' preference only approached significance $(p=.07)$. For the combined-species sample, there was a reliable choice of Grammatical over Nongrammatical elements.

The phrase "learning something about the grammar" requires comment. In the context of the artificial grammar used in this study, learning something about the grammar might mean nothing more than that the subject had learned some or all of the grammatically correct transitions between the elements that were experienced in training. As a result, at the time of the grammatical/nongrammatical pair-wise test, the subject might have been choosing between a transition it had experienced previously, although not at precisely that point in the chain of 128 elements, and one it had not previously seen. In this sense, some involvement of the specific learning of these transitions cannot be ruled out, although it should be kept in mind that in this procedure, such learning was not required. The procedure allowed the subject to adopt a simpler rule, such as "Touch the image wherever it occurs and periodically food comes." If this analysis is correct, it indicates that learning beyond what was strictly required by the contingencies of the experiment took place, although to what extent subjects may have appreciated the overall structure of the grammar apart from learning specific grammatical transitions between the elements cannot be determined (see also Eitam, Kennedy, \& Higgins, 2013, for possible additional sources of motivation/incentive in this kind of procedure). It should be added that, as we previously noted, the criticism that what was actually acquired was knowledge of specific transitions and not some holistic understanding of the grammar could also be made of Reber's original work (see Brooks \& Vokey, 1991, for a similar criticism).

The last comparison, between Next and Grammatical elements, carried with it interpretative difficulties as well, because both elements were grammatical, although Next elements could also be chosen on the basis of memorization of the sequence. The tamarins reliably chose Next over Grammatical elements, although this preference was principally driven by Homer's strong preference for Next over Grammatical elements. The remaining two tamarins were indifferent on this choice test. The pigeons also demonstrated indifference on this choice test. Overall, then, five of the six subjects were indifferent on this choice test. Indifference is itself difficult to interpret, because indifference could result from a lack of learning the grammar, although the random test and the Next-versus-Nongrammatical pair-wise test results argue against a failure to learn. Indifference may also result from subjects having acquired some appreciation of the grammar and responding on that basis, rather than based on memorization of the overall sequence. For the combined-species sample, the preference for Next elements approached significance, although this preference was again driven principally by Homer's strong choice of Next over Grammatical elements.

It should be pointed out that this study was not the first in which we observed that the pair-wise test results were not as clearly interpretable as were the results of the random test, or as the results generated in standard studies of serial learning. Locurto et al. (2009), for example, noted that there appeared to be less precise control over internal pair choices (e.g., B/D) in an implicit-chaining procedure than is evident in standard studies of serial learning, in which reinforcement is delivered for correct responses (cf. Fig. 2 of Locurto et al., 2009, with Terrace \& McGonigle, 1994). In interpreting this feature of these results, we underscore the point that the random test, derived from the implicit-learning literature, is an embedded test that does not interrupt the ongoing task. In contrast, the pair-wise tests represented the first time in training that the chain was interrupted and the subject was asked to choose between stimuli. In more standard serial learning tasks, the subject is well adapted to choosing between paired stimuli during the course of training.

Additionally, to retain the implicit nature of the task, reinforcement was delivered randomly following pair-wise test choices in this study. In standard serial learning experiments, reinforcement is delivered only following correct responses. The combination of these factors may have resulted in the pair-wise tests being less sensitive measures of what was learned in this design than they are in more standard serial learning tasks. With that caveat in mind, subjects in both species nonetheless chose Next over Nongrammatical elements and Grammatical over Nongrammatical elements. With the exception of Homer, all of the subjects also demonstrated indifference between Next and Grammatical elements.

This pattern of results, in which the performances of tamarins and pigeons were similar in some respects (random test, pair-wise tests) and reliably different in others (baseline performance), mirrors the literature in which primate and avian species have been compared on cognitive tasks. As we cited previously, there are some similarities and some notable differences between primates and pigeons in serial learning and in transitive inference tasks. The present results may be situated within that portion of the literature in which comparable between-species performance has been observed. The finding of similarities between tamarins and pigeons on an implicit 
learning task using an artificial grammar should perhaps be less unexpected now than it might have been previously. Part of the reason for this lack of surprise is precisely the growing literature that has indicated comparable performance between primates and avians. This literature articulates with the modern recognition that the avian brain enjoys more similarities with the mammalian brain than may have previously been understood (Colombo \& Scarf, 2012; Shanahan, Bingman, Shimizu, Wild, \& Güntürkün, 2013; Shimizu, 2009). In the past, these parallels may have been unforeseen, given the understanding that mammals have been evolving separately from birds for nearly 300 million years. The appreciation of these similarities in performance, whatever their evolutionary source, whether they result from convergence or common descent, has made avians suitable for a wider variety of cognitive research than perhaps has previously been suspected, including the use of songbirds as a model for human language evolution (Berwick, Beckers, Okanoya, \& Bolhuis, 2012; Lefebvre, Reader, \& Sol, 2004).

Author note This study was supported by NIH Grant No. 1 R15RR031220-01A1. We thank the New England Primate Research Center, Southborough, MA, for supporting the work with cotton-top tamarins.

\section{References}

Becker, L. A. (2000, March 21). Effect size calculators. Retrieved January 16, 2014, from www.uccs.edu/lbecker/index.html

Berwick, R. C., Beckers, G. J. L., Okanoya, K., \& Bolhuis, J. J. (2012). A bird's eye view of human language evolution. Frontiers in Evolutionary Neuroscience, 4, 5. doi:10.3389/fnevo.2012.00005

Bond, A. B., Kamil, A. C., \& Balda, R. P. (2003). Social complexity and transitive inference in corvids. Animal Behaviour, 65, 479-487. doi: 10.1006/anbe. 2003.2101

Brannon, E. M., \& Terrace, H. S. (2002). The evolution and ontogeny of ordinal numerical ability. In M. Bekoff, C. Allen, \& G. M. Burghardt (Eds.), The cognitive animal: Empirical and theoretical perspectives on animal cognition (pp. 197-204). Cambridge, MA: MIT Press.

Brooks, L. R., \& Vokey, J. R. (1991). Abstract analogies and abstracted grammars: Comments on Reber (1989) and Mathews et al. (1989). Journal of Experimental Psychology: General, 120, 316-323. doi: 10.1037/0096-3445.120.3.316

Bulmer, M. G. (1979). Principles of statistics. New York, NY: Dover.

Chen, S., Swartz, K. B., \& Terrace, H. S. (1997). Knowledge of the ordinal position of list items in rhesus monkeys. Psychological Science, 8, 80-86. doi:10.1111/j.1467-9280.1997.tb00687.x

Chomsky, N. (1957). Syntactic structures. Oxford, UK: Mouton.

Christie, M. A., \& Dalrymple-Alford, J. C. (2004). A new rat model of the human serial reaction time task: Contrasting effects of caudate and hippocampal lesions. Journal of Neuroscience, 24, 1034-1039. doi: 10.1523/JNEUROSCI. 3340-03.2004

Christie, M. A., \& Hersch, S. M. (2004). Demonstration of nondeclarative sequence learning in mice: Development of an animal analog of the human serial reaction time task. Learning and Memory, 11, 720723. doi:10.1101/lm. 85404
Clegg, B. A., DiGirolamo, G. J., \& Keele, S. W. (1998). Sequence learning. Trends in Cognitive Sciences, 2, 275-281. doi:10.1016/S13646613(98)01202-9

Colombo, K., \& Scarf, D. (2012). Neurophysiological studies of learning and memory in pigeons. Comparative Cognition \& Behavior Reviews, 7, 23-43. doi:10.3819/ccbr.2012.70002

Colombo, M., Cottle, A., \& Frost, N. (2003). Degree of representation of the matching concept in pigeons (Columba livia). Journal of Comparative Psychology, 117, 246-256. doi:10.1037/0735-7036. 117.3.246

D'Amato, M. R. (1991). Comparative cognition: Processing of serial order and serial pattern. In L. Dachowski \& C. F. Flaherty (Eds.), Current topics in animal learning: Brain, emotion, and cognition (pp. 165-185). Hillsdale, NJ: Erlbaum.

D'Amato, M. R., \& Colombo, M. (1988). Representation of serial order in monkeys (Cebus apella). Journal of Experimental Psychology: Animal Behavior Processes, 14, 131-139. doi:10.1037/0097-7403. 14.2.131

D'Amato, M. R., \& Colombo, M. M. (1990). The symbolic distance effect in monkeys (Cebus apella). Animal Learning \& Behavior, 18, 133-140. doi:10.3758/BF03205250

D’Amato, M. R., Salmon, D. P., Loukas, E., \& Tomie, A. (1986). Processing of identity and conditional relations in monkeys (Cebus apella) and pigeons (Columba livia). Animal Learning \& Behavior, 14, 365-373. doi:10.3758/BF03200080

Davis, H. (1992). Transitive inference in rats (Rattus norvegicus). Journal of Comparative Psychology, 106, 342-349. doi:10.1037/07357036.106.4.342

Deroost, N., \& Soetens, E. (2006). Perceptual or motor learning in SRT tasks with complex sequence structures. Psychological Research, 70, 88-102. doi:10.1007/s00426-004-0196-3

Dienes, Z., \& Berry, D. (1997). Implicit learning: Below the subjective threshold. Psychonomic Bulletin \& Review, 4, 3-23. doi:10.3758/ BF03210769

Domenger, D., \& Schwarting, R. W. (2005). Sequential behavior in the rat: A new model using food-reinforced instrumental behavior. Behavioural Brain Research, 160, 197-207. doi:10.1016/j.bbr. 2004.12.002

Eitam, B., Kennedy, P. M., \& Higgins, E. (2013). Motivation from control. Experimental Brain Research, 229, 475-484. doi:10.1007/ s00221-012-3370-7

Frensch, P. A., \& Rünger, D. (2003). Implicit learning. Current Directions in Psychological Science, 12, 13-18. doi:10.1111/1467-8721.01213

Froehlich, A. L., Herbranson, W. T., Loper, J. D., Wood, D. M., \& Shimp, C. P. (2004). Anticipating by pigeons depends on local statistical information in a serial response time task. Journal of Experimental Psychology: General, 133, 31-45. doi:10.1037/0096-3445.133.1.31

Herbranson, W. T., \& Shimp, C. P. (2008). Artificial grammar learning in pigeons. Learning \& Behavior, 36, 116-137. doi:10.3758/LB.36.2. 116

Herbranson, W. T., \& Stanton, G. L. (2011). Flexible serial response learning by pigeons (Columba livia) and humans (Homo sapiens). Journal of Comparative Psychology, 125, 328-340. doi:10.1037/ a0023299

Kenny, D. T. (1953). Testing of differences between variances based on correlated variates. Canadian Journal of Psychology, 7, 25-28. doi: $10.1037 / \mathrm{h} 0083569$

Killeen, P. R. (2003). Complex dynamic processes in sign tracking with an omission contingency (negative automaintenance). Journal of Experimental Psychology: Animal Behavior Processes, 29, 49-61. doi:10.1037/0097-7403.29.1.49

Knowlton, B. J., \& Squire, L. R. (1994). The information acquired during artificial grammar learning. Journal of Experimental Psychology: Learning, Memory, and Cognition, 20, 79-91. doi:10.1037/02787393.20.1.79 
Lefebvre, L., Reader, S. M., \& Sol, D. (2004). Brains, innovations and evolution in birds and primates. Brain, Behavior and Evolution, 63, 233-246. doi:10.1159/000076784

Locurto, C., Dillon, L., Collins, M., Conway, M., \& Cunningham, K. (2013). Implicit chaining in cotton-top tamarins (Saguinus oedipus) with elements equated for probability of reinforcement. Animal Cognition, 16, 611-625. doi:10.1007/s10071-013-0598-y

Locurto, C., Gagne, M., \& Levesque, K. (2009). Implicit chaining in cottontop tamarins (Saguinus oedipus). Journal of Experimental Psychology: Animal Behavior Processes, 35, 116-122. doi:10.1037/a0012924

Locurto, C., Gagne, M., \& Nutile, L. (2010). Characteristics of implicit chaining in cotton-top tamarins (Saguinus oedipus). Animal Cognition, 13, 617-629. doi:10.1007/s10071-010-0312-2

Locurto, C. M., Terrace, H. S., \& Gibbon, J. (1981). Autoshaping and conditioning theory. New York, NY: Academic Press.

Marcus, G. F., Vijayan, S., Rao, S. B., \& Vishton, P. M. (1999). Rule learning by seven-month-old infants. Science, 283, 77-80. doi:10. $1126 /$ science. 283.5398 .77

Merritt, D. J., \& Terrace, H. S. (2011). Mechanisms of inferential order judgments in humans (Homo sapiens) and rhesus monkeys (Macaca mulatta). Journal of Comparative Psychology, 125, 227-238. doi: 10.1037/a0021572

Meyer, T., \& Olson, C. R. (2011). Statistical learning of visual transitions in monkey inferotemporal cortex. Proceedings of the National Academy of Sciences, 108, 19401-19406. doi:10.1073/pnas.1112895108

Nissen, M. J., \& Bullemer, P. (1987). Attentional requirements of learning: Evidence from performance measures. Cognitive Psychology, 19, 1-32. doi:10.1016/0010-0285(87)90002-8

Nissley, H. M., \& Schmitter-Edgecombe, M. (2002). Perceptually based implicit learning in severe closed-head injury patients. Neuropsychology, 16, 111-122. doi:10.1037/0894-4105.16.1.111

Procyk, E., Dominey, P., Amiez, C., \& Joseph, J. (2000). The effects of sequence structure and reward schedule on serial reaction time learning in the monkey. Cognitive Brain Research, 9, 239-248. doi:10. 1016/S0926-6410(00)00002-1

Rapp, P. R., Kansky, M. T., \& Eichenbaum, H. (1996). Learning and memory for hierarchical relationships in the monkey: Effects of aging. Behavioral Neuroscience, 110, 887-897. doi:10.1037/07357044.110.5.887

Reber, A. S. (1967). Implicit learning of artificial grammars. Journal of Verbal Learning and Verbal Behavior, 6, 855-863. doi:10.1016/ S0022-5371(67)80149-X

Reber, A. S. (1993). Implicit learning and tacit knowledge: An essay on the cognitive unconscious. New York, NY: Oxford University Press.

Roberts, W. A. (2010). "Counting" serially presented stimuli by human and nonhuman primates and pigeons. Learning and Motivation, 41, 241-251. doi:10.1016/j.lmot.2010.08.002

Roberts, W. A., \& Mazmanian, D. S. (1988). Concept learning at different levels of abstraction by pigeons, monkeys, and people. Journal of Experimental Psychology: Animal Behavior Processes, 14, 247 260. doi:10.1037/0097-7403.14.3.247

Saffran, J. R., Aslin, R. N., \& Newport, E. L. (1996). Statistical learning by 8-month-old infants. Science, 274, 1926-1928. doi:10.1126/ science. 274.5294 .1926

Saffran, J. R., \& Thiessen, E. D. (2003). Pattern induction by infant language learners. Developmental Psychology, 39, 484-494. doi: 10.1037/0012-1649.39.3.484

Scarf, D., \& Colombo, M. (2008). Representation of serial order: A comparative analysis of humans, monkeys, and pigeons. Brain Research Bulletin, 76, 307-312. doi:10.1016/j.brainresbull.2008.02.022
Scarf, D., Hayne, H., \& Colombo, M. (2011). Pigeons on par with primates in numerical competence. Science, 334, 1664-1667. doi:10. 1126/science. 1213357

Seger, C. (1994). Implicit learning. Psychological Bulletin, 115, 163-196. doi:10.1037/0033-2909.115.2.163

Shanahan, M., Bingman, V. P., Shimizu, T., Wild, M., \& Güntürkün, O. (2013). Large-scale network organization in the avian forebrain: A connectivity matrix and theoretical analysis. Frontiers of Computational Neuroscience, 7, 89. doi:10.3389/fncom.2013.00089

Shimizu, T. (2009). Why can birds be so smart? Background, significance, and implications of the revised view of the avian brain. Comparative Cognition and Behavior Reviews, 4, 103-115. doi: 10.3819/ccbr.2009.40011

Smith, J., Berg, M. E., Cook, R. G., Murphy, M. S., Crossley, M. J., Boomer, J., \& Grace, R. C. (2012). Implicit and explicit categorization: A tale of four species. Neuroscience \& Biobehavioral Reviews, 36, 2355-2369. doi:10.1016/j.neubiorev.2012.09.003

Soetens, E. E., Melis, A. A., \& Notebaert, W. W. (2004). Sequence learning and sequential effects. Psychological Research, 69, 124-137. doi:10.1007/s00426-003-0163-4

Straub, R. O., \& Terrace, H. S. (1981). Generalization of serial learning in the pigeon. Animal Learning \& Behavior, 9, 454-468.

Terrace, H. S. (1993). The phylogeny and ontogeny of serial memory: List learning by pigeons and monkeys. Psychological Science, 4, 162-169. doi:10.1111/j.1467-9280.1993.tb00481.x

Terrace, H. S. (2001). Chunking and serially organized behavior in pigeons, monkeys and humans. In R. G. Cook (Ed.), Avian visual cognition. Medford, MA: Comparative Cognition Press. Retrieved from www.pigeon.psy.tufts.edu/avc/terrace/

Terrace, H. (2010). The comparative psychology of serially organized behavior. Comparative Cognition \& Behavior Reviews, 5, 23-58. doi:10.3819/ccbr.2010.50002

Terrace, H. S., \& McGonigle, B. (1994). Memory and representation of serial order by children, monkeys, and pigeons. Current Directions in Psychological Science, 3, 180-189. doi:10.1111/1467-8721. ep10770703

Weaver, J. E., Steirn, J. N., \& Zentall, T. R. (1997). Transitive inference in pigeons: Control for differential value transfer. Psychonomic Bulletin \& Review, 4, 113-117. doi:10.3758/BF03210782

Williams, H., \& Staples, K. (1992). Syllable chunking in zebra finch (Taeniopygia guttata) song. Journal of Comparative Psychology, 106, 278-286. doi:10.1037/0735-7036.106.3.278

Wright, A. A., Santiago, H. C., Sands, S. F., Kendrick, D. F., \& Cook, R. G. (1985). Memory processing of serial lists by pigeons, monkeys, and people. Science, 229(4710), 287-289. doi:10.1126/science. 9304205

Wright A. A., \& Katz, J. S. (2007). Generalization hypothesis of abstractconcept learning: Learning strategies and related issues in Macaca mulatta, Cebus apella, and Columba livia. Journal of Comparative Psychology, 121, 387-397. doi:10.1037/0735-7036.121.4.387

Wright, A. A., \& Katz, J. S. (2009). A case for restricted-domain relational learning. Psychonomic Bulletin \& Review, 16, 907-913. doi: 10.3758/PBR.16.5.907

Wuensch, K. L. (2012). Nonparametric effect size estimators. Retrieved January 16, 2014, from http://core.ecu.edu/psyc/wuenschk/docs30/ Nonparametric-EffectSize.docx

Wynne, C. L. (1997). Pigeon transitive inference: Tests of simple accounts of a complex performance. Behavioural Processes, 39, 95112. doi:10.1016/S0376-6357(96)00048-4

Yamazaki, Y., Suzuki, K., Inada, M., Iriki, A., \& Okanoya, K. (2012). Sequential learning and rule abstraction in Bengalese finches. Animal Cognition, 15, 369-377. doi:10.1007/s10071-011-0462-x 